\title{
Reliable Multipath Multi-Channel Route Migration over Multi Link-Failure in Wireless Ad hoc Networks
}

\author{
Nawaf S. Mirza*, Peter J. B. King*, Imed Romdhani ${ }^{\dagger}$, Mohamed A. Abdelshafy*, Atif A. Alghamdi * \\ ${ }^{*}$ School of Mathematical and Computer Sciences \\ Heriot-Watt University, Edinburgh, UK \\ \{nm246, P.J.B.King, m.abdelshafy, aaa148\}@hw.ac.uk \\ ${ }^{\dagger}$ School of Computing, Edinburgh Napier University, Edinburgh, UK \\ \{I.Romdhani\}@napier.ac.uk
}

\begin{abstract}
The route recovery algorithm is a crucial part of an ad hoc routing protocol. Designing an efficient and fast route recovery mechanism scheme without incurring extra overheads or delays to repair the broken link is a desirable goal for any routing protocol. The route recovery algorithm in reactive routing protocols like AODV occurs only after a link failure is detected. However, we propose a new route discovery mechanism called Route Migration over Multiple link failure in Multi-Channel (RMMMC), which combines multiple paths and channels to provide a disjointed alternative route. When link failure is detected, the RMMMC reroutes the data packet through the alternative route in alternative channels that have been previously discovered. Furthermore, the RMMMC is resilient to multiple link failures. Unlike other route recovery approaches, nodes in RMMMC are considered to be equipped with a single transceiver. The RMMMC scheme is implemented and extensively evaluated by using an ns-2 simulator. Simulation results show that the proposed scheme achieves a better packet delivery ratio and throughout in a dense network.
\end{abstract}

Keywords- Route discovery, Route Recovery, Multipath, Multichannel wireless network, Link Failure, AODV

\section{INTRODUCTION}

A Mobile Ad hoc Network (MANET) is a collection of mobile nodes which form a self-configurable and selforganising network without relying on any fixed infrastructure. Mobile nodes in MANET communicate directly, if they are in the same transmission range, while those which are not can use other nodes to relay their packets in a multi-hop manner. Due to the unique characteristics of wireless channel, such as interference, low bandwidth and contention in radio channels, communication links in MANETs are considered unreliable.

Wireless ad hoc routing protocols are responsible for discovering a route to a destination and handling a link failure in the path. Such protocols will provide a high packet delivery ratio and utilise the network resources efficiently. Wireless ad hoc protocols such AODV [1] and DSR [2] deploy two mechanisms to detect link failure, namely, a Hello message and link layer feedback. Such mechanisms enable nodes to obtain an immediate knowledge to verify that the next-hop is within their transmission range and is likely to receive the packet. Consequently, network resources and bandwidth will not be wasted.
A Hello message is periodically broadcast every time interval to maintain local connectivity with one-hop neighbours. Failure to receive a Hello message for several successive time intervals, indicates that the link to this neighbour has failed and it is no longer within transmission range [3]. Hence, the node will start a local repair procedure, if possible. The second mechanism to detect link failure is link layer feedback. In this approach, node can identify the link failure during transmission of a data packet to another node by the absence of a link layer acknowledgement message. For instance, the MAC layer protocol used to transmit unicast packets in IEEE 802.11 DCF [4], uses RTS and CTS control packets. The absence of CTS or ACK control packets initiates a retransmission procedure. If the retransmission retries reaches the allowed retries limit, the node will assume that the link with this node has broken and, thus, will send a feedback message to the higher layer to take an appropriate action.

Utilising multiple non-overlapping frequency channels can improve network capacity by enabling adjacent nodes to transmit simultaneously in different channels. Wireless communication standards such as IEEE $802.11 \mathrm{a} / \mathrm{b}$ [4] offer 3 and 12 non-overlapping channels respectively. Hence, a new routing protocol is necessary to utilise these channels and to consider solutions for link failure issues.

Therefore, a reliable routing protocol called Route Migration over Multiple link failure in Multi-Channel with a single radio interface (RMMMC) is proposed. It introduces a new route discovery mechanism, which utilises multiple channels to provide a seamless alternative multi-hop path when link failure is detected. RMMMC can handle multiple link failures in the route. The protocol utilises the concept of a Receiver Directed Transmission (RDT-AODV) protocol [5] with a single radio interface to implement the multi-channel network and the reactive routing protocol (AODV)[1] as a means to implementing RMMMC.

The rest of this paper is organized as follows. In Section II, we review some related work. In Section III identifies the problems to be addressed. In section IV, our schemes are proposed, while Section V presents the performance evaluation and simulation results. Section VI presents the performance results and, finally, Section VII summarizes key results and issues. 


\section{RELATED WORK}

In this section, we provide a brief review of some of the multipath routing protocols based on AODV, and then briefly describe the multichannel routing protocol used in this work.

AODV is a reactive routing protocol which discovers a route only on a single path when needed. AODV uses messages such as Route Request (RREQ), Route Reply (RREP) and Route Error (RERR) to discover a routing path to the destination. RERR is used to establish a local repair if the routing path is disconnected.

Many on-demand multi-path routing protocols have been proposed for mobile ad hoc networks, which include the Ad hoc On- demand Multipath Distance Vector (AOMDV) [6] and Ad hoc On-demand Distance Vector Backup Routing (AODV$\mathrm{BR}$ ) [7]. In AODV-BR, an overhearing mechanism is used to construct a secondary path. More specifically, neighbour nodes which overhear the RREP packet will make themselves act as a secondary path to this destination. When link failure is detected, the node will broadcast data packets to all neighbour nodes. A node that receives the data packet will forward it to its secondary path, which is constructed by overhearing the RREP. Thus, AODV-BR is not truly a multipath extension of AODV, as nodes have just one routing path to any destination.

AOMDV is a link-disjoint multipath routing protocol based on AODV. Unlike AODV, the AOMDV protocol stores all received RREQ packets. A new field in the RREQ packet, called firsthop, indicates the neighbour node of the source nodes. So, when node receives the RREQ packet, it will compare the firsthop field in the RREQ with its own firsthop_list in the routing table. If the RREQ contains a different firsthop, then the node will store the new next hop information to its routing table for multiple routing paths. In contrast to AODV, AOMDV enables the destination node to send a RREP for each RREQ packet received. Thus multiple routes between source and destination are formed. AOMDV considers multiple paths over a single wireless channel only.

Several researchers have proposed routing and channel assignment protocols for multichannel wireless communication [8], [9], [5]. So et al. [9] proposed the Multi-Channel Routing Protocol (MCRP), which utilises multiple channels to improve the network capacity with the aid of a single transceiver only. It improves network performance by trying to allocate different channels to different flows; thus, it will enable a simultaneous transmission among adjacent nodes. MCRP assigns a common channel to all nodes in the flow and uses LEAVE/JOIN messages to eliminate the deafness problem. MCRP considers a single path solution only and used force mechanism to avoid deafness problem.

Mirza et al. [5] proposed the Receiver-Directed Transmission protocol (RDT-AODV) to utilise multiple channels. In RDT-AODV each node is equipped with a single half-duplex transceiver. RDT-AODV facilitates channel assignment and negotiation without using a control channel or time synchronisation. In RDT-AODV, each node is assigned a home channel to which the node is listening when not transmitting, by using the following equation [5]:

$$
\left(H \_C h=N \_I d \bmod T \_C h\right)(1)
$$

where $H \_C h$ is nodes home channel, $N_{-} I d$ is node IP address and $T_{-} \bar{C} h$ is the total number of available channels in the network. The home channel for any node in the network can be calculated using equation (1). Let us assume that node $S$ has data to send to node $D$, as in Fig 1. Firstly, node $S$ will calculate and switch its channel to the destination's home channel for this transmission only by equation (1). Secondly, it will broadcast a RREQ packet in the destination home channel. From neighbours in the destination home channel, the packet will be routed until it reaches its destination. When RREQ reaches node $D$, it will unicast a RREP on the next hop node's home channel, and so on, until the packet reaches node $S$. RDT-AODV assumes that nodes are more likely to find at least one neighbour operating in every channel in a dense network.

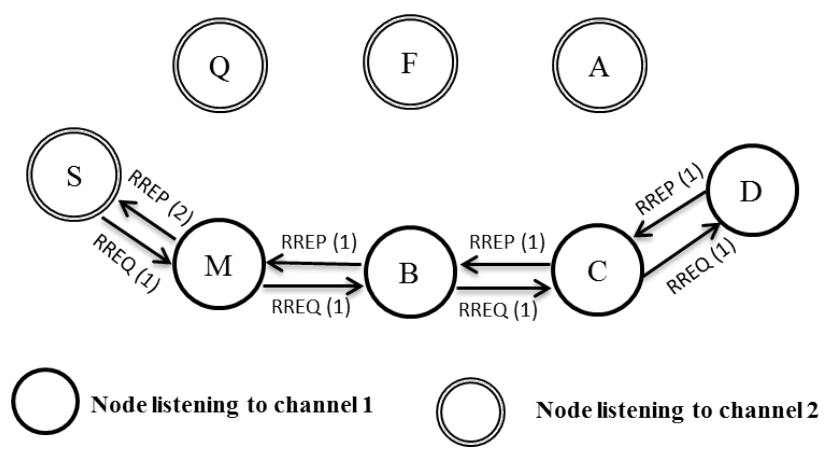

Fig. 1: Route Discovery process in RDT-AODV.

\section{PROBlem STATEMENT}

This section will summarise the problems addressed by the proposed protocol and address the following issues related to the multichannel RDT-AODV and AODV routing protocols.

Although the RDT-AODV communication scheme facilitates implementing a multi-channel network with a single transceiver, it does not provide enough flexibility, as it uses a deterministic channel assignment approach. For example, communication might not be established if there is no neighbour node operating in the destination home channel, which wastes the network resources.

In the RDT-AODV protocol, each node is equipped with a single transceiver and transmits in the destination home channel. Hence, the intended receiver of a packet may be switched to another channel handling different transmissions. In such a case, the receiver node is deaf during this time and before it moves back to its channel, the transmitter node will assume the link has broken and will send a link layer feedback to higher layer and invalidate the route.

Furthermore, a local repair algorithm in AODV is used to repair the broken link only if the failure occurs closer to the destination than to the source node. In this case, the node will attempt to repair the route by broadcasting a RREQ packet with a limited Time-To-Live. Otherwise, the node will drop the data packet, invalidate the route and send a Route Error (RERR) message to the source node, which will initiate another route discovery process and flood the network again, causing extra overhead and delay. 
The proposed protocol deals with the above issues by introducing a new routing discovery mechanism which increases the probability of discovering a route to the destination, even when no neighbour is operating in the destination home channel. Furthermore, when a node is deaf or the link has broken, it will re-route the data packet through the alternative route in an alternative channel which has already been discovered. Moreover, the proposed scheme can repair multiple link failures, wherever their location, without incurring extra overhead or delay.

\section{PROPOSED PROTOCOL}

The proposed protocol is designed for a multi-failure, multipath route recovery in a highly dense, dynamic, multichannel network. It is based on the Multi-Channel ReceiverDirected Transmission (RDT-AODV) protocol [5]. The nodes are assumed to be equipped with a single half-duplex radio interface, as most of the current wireless devices are.

The proposed protocol introduces a new routing mechanism, which occurs over multiple paths in multiple channels in order to provide an alternative path. When link failure occurs, the node will re-route (handover) the data packets between different established paths by selecting the nearest neighbour from the alternative path. Thus, it will improve the packet delivery ratio and reduce the overhead and end-to-end delay caused by route re-discovery.

The proposed scheme will be presented in detail in the following sections.

\section{A. Local Connectivity in RMMMC protocol:}

RMMMC is proposed to improve the network capacity in a multichannel RDT-AODV protocol by providing a multipath solution to link failure problems. In RDT-AODV, every node listens to its own home channel while not transmitting a data packet. Hence, the node will not be aware of its neighbour operating in different channels, which may cause a route disconnection and waste of network resources.

However, in the proposed protocol, we have modified the Hello message procedure to enable nodes to broadcast Hello packets in all available channels in a Round Robin manner. In Figure 2, let us assume that there are three available channels in the network. Therefore, node 2 broadcasts a Hello message in channel 1 in the first interval; then it will broadcast a Hello message in channel 2 in the next interval, and so on. This will enable a node to inter-connect with its neighbours operating in different channels.

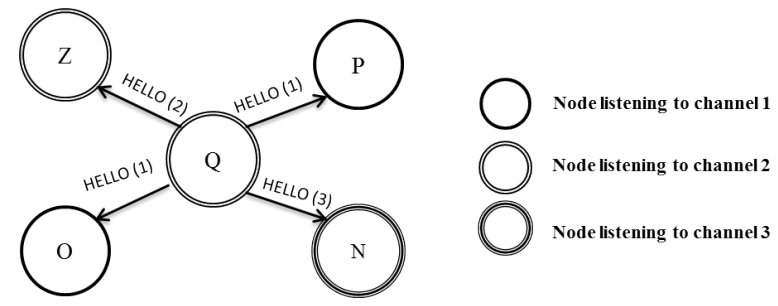

Fig. 2: Hello packet in RMMMC.

\section{B. Routing Tables}

To implement RMMMC, we introduced a few changes to the routing table, RREQ and RREP packet frame formats. Furthermore, a new route control packet called the MultiHop PAth packet (MHPA) is introduced to provide a full multi-hop alternate path in different channel. This packet will create/update a route entry in a new routing table (alternate routing table) which is used to re-route the data packet when link failure occurs.

In the routing table, the following fields were added:

- rt_accumulated_no: which contains the number of addresses in the accumulated path.

- rt_pri_path[]: which contains an accumulated address for any routing entry.

\section{Alternative Routing Table:}

The RMMMC proposes a new routing table called the alternative table to enable a node to re-route the data packet when link failure is detected. In RMMMC, an ageing technique is used for alternate route maintenance, which is similar to the original AODV protocol.

The alternative routing table contains the following information:

- Destination

- Next hop

- Hop count

- Destination sequence no

- Expiration time

- al_accumulated_no: which contains the number of addresses in the accumulated path.

- al_backup_path[]: which contains an accumulated addresses for the backup route.

A node will create/update its alternative routing table in the following cases:

1) Destination node: when destination node receives RREQ/RREP with BACKUP flag.

2) Node receives MHPA packet.

\section{Control Packets}

\section{Route Request and Route Reply packets:}

Considering the original AODV and RDT-AODV protocols, which do not support route accumulation, the following changes have been made to the RREQ/RREP packets to support route accumulation:

- accumulated_no: which is the number of accumulated addresses.

- accumulated_path[]: which contains the accumulated addresses.

- flags: to distinguish between RREQ/RREP sent in original (original) and alternative (backup) routes.

RMMMC uses the reserved fields in RREQ/RREP packets to implement accumulated_no and flags fields and add accumulated_path as a new field. Furthermore, the RREQ/RREP 
packet size has been modified to dynamically reflect the actual size of the packet which depends on the number of addresses in the accumulated_path[].

\section{Multi-Hop PAth packet(MHPA):}

RMMMC introduces a new control packet called the MHPA packet. The format of the MHPA packet is similar to the above RREP packet, but it has different functionality. The MHPA packet will only be sent by the destination node when it has two entries for any destination (an entry in the routing table and an entry in the alternative table). Furthermore, it is used to announce the possibility of an alternative multi-hop path in a different channel. The MHPA packet is a destinationoriented packet which is used to create/update the alternative routing table only.

\section{Route Discovery mechanism}

Unlike AODV, RMMMC introduces a new routing discovery mechanism by initialising multiple route discovery processes for any destination and support route accumulation. For simplicity, we consider two route discovery process for each destination.

\section{1) Route Accumulation:}

RMMMC requires route accumulation in order to provide a full multi-hop alternative path to cope with link failure. Hence, certain changes have been introduced to enable the node to include and accumulate its addresses before transmitting the RREQ/RREP packet. This will provide a multi-hop path for any entry in the routing table. For instance, in Figure 3, the source node $S$ will include its address in the accumulation_path[] before broadcasting the RREQ. Nodes $M$ and $Q$ will receive the RREQ in their home channel and will create a route entry to node $S$ with the accumulated path $\{S\}$. Then nodes $M$ and $Q$ will include their own addresses in the accumulated_path[] and re-broadcast the RREQ in their home channel, and so on. The same concept of route accumulation is applied to the RREP packet. Therefore, each node will acquire the multi-hop path between itself and any destination in its routing table.

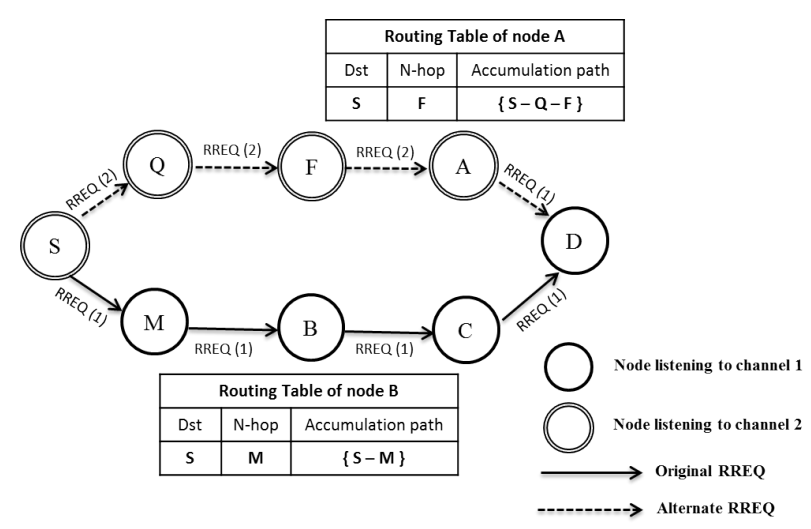

Fig. 3: Route Request process in RMMMC.

\section{2) Broadcasting Route Request packet:}

In RMMMC, the source node will broadcast two RREQ packets in two different channels. The first RREQ (original route) will be in the Destination Home Channel (D'HC), as in the RDT-AODV protocol, while the second RREQ (alternative route) will be sent in a different channel. All nodes in the original and alternative routes will broadcast the RREQ in their own home channel, with the exception of when a node is in the alternative route and the final destination is in its neighbour table. In this case, the node will switch its channel to D'HC and broadcast the RREQ. This is required to enable the destination node to receive and respond to the RREQ. For instance, in Figure 3 , nodes $M-B-C-Q-F$ will rebroadcast the RREQ in their own home channels. However, node $A$ will switch its channel and rebroadcast the RREQ in node $D^{\prime} s$ home channel, as it finds that node $D$ is in its neighbour table.

Nodes receiving the RREQ packet will create/update their routing entry to node $S$ in their routing table. However, the destination node will create/update its routing entry based on the RREQ type (original or alternative). If the RREQ was sent in its own home channel (original flag), then it will create/update its routing table. Otherwise, it will create/update its alternative routing table.

The benefit of broadcasting two RREQ packets -can be more- is to increase the probability of discovering a route to a destination when none of the neighbours is operating in the destination home channel. Additionally, it is able to provide a seamless handover multi-hop alternate route in a different channel when link failure occurs.

3) Sending Route Reply packet:

The Route Reply (RREP) packet will be unicast in the reverse route when the destination node or an intermediate node with a fresh route to the destination receives the RREQ. As there are two RREQs broadcast in two channels, there will be two RREP packets unicast to the source node in two different channels. Thus, the node may discover multiple paths to any destination node. The RMMMC will use the route accumulation mechanism as described above and will send the RREP packet as follows:

- Destination node: When the destination node receives the RREQ it will update its corresponding table depending on the RREQ flag and then send the RREP to the source node via the node which broadcast the RREQ. Thus, in Figure 4, node $D$ will send an RREP via node $C$ in channel 1 and another RREP via node $A$ in channel 2 .

- Intermediate node. When an intermediate node with a fresh route to the destination receives the RREQ, it will send an RREP to source node with its accumulated path to the destination.

In order to enable the destination node to acquire the full multi-hop path to any node, we enable the intermediate node to send a 
gratuitous RREP packet. Therefore, the intermediate node will also send the RREP to the destination node with the accumulated path to the source node.

Let us assume node $B$ in Figure 4 receives a RREQ from $S$ and has a fresh route to node $D$. In this case, $B$ will send a RREP to $S$, including its accumulated path to $D\{D, C, B\}$. It will also send a gratuitous RREP to $D$ with the accumulated path to $S\{S, M, B\}$.

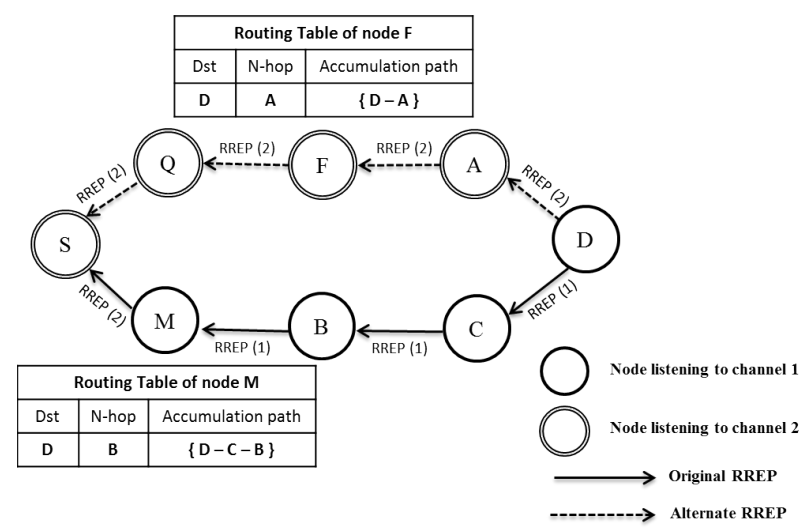

Fig. 4: Route Reply process in RMMMC.

\section{4) Sending MHPA packet:}

When a destination node has two entries to any destination entry (one entry in the routing table and one entry in the alternative table), then it will send an MHPA packet to inform the intermediate nodes about the possible alternative route in a different channel when a link failure occurs.

Figure 5 shows the process of sending an MHPA packet in RMMMC. Let us assume the destination node $D$ has built two full multi-hop paths towards node $S$, namely, path $1\{S, M, B, C\}$ in its routing table and path $2\{S, Q, F, A\}$ in its alternate table. In this case, node $D$ will send an MHPA packet to node $S$ : First, node $D$ will apply the Least Hop First Algorithm (LHF) [10] to both paths, in order to enable the intermediate node detecting the link failure to select the least hop count to itself first. So, path 1 will be $\{C, B, M, S\}$ and path $2\{A, F, Q, S\}$. Second, node $D$ will unicast the two MHPA packets to node $S$ in two different routes. Path 1 will be unicast to the next hop in the opposite route (node $A)$ and path 2 will be unicast to the next hop in the opposite route $(\operatorname{node} C)$. Nodes which receive the MHPA packet will update their alternative route only and then forward the packet to their next hop in the routing table toward the destination. Consequently, intermediate nodes will acquire the full multi-hop path of an alternative route. Node $D$ will send a MHPA whenever it gets a fresher route to any destination in its tables. This is important to maintain an up-to-date multi-hop alternative in the intermediate node.

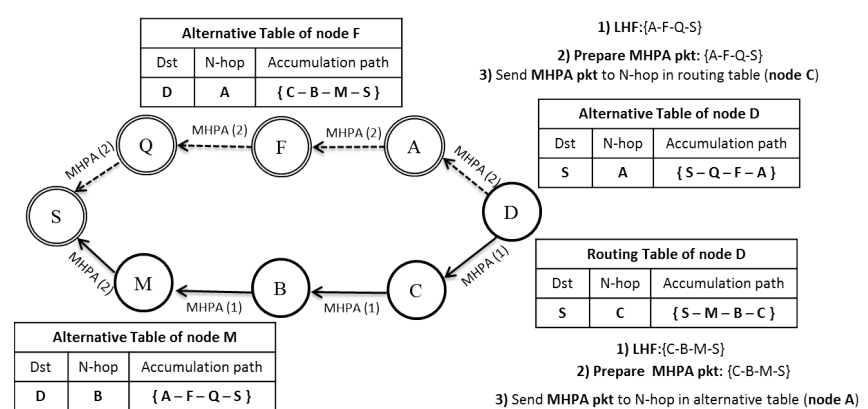

Fig. 5: Sending Multi-Hop PAth packet (MHPA) in RMMMC.

\section{E. Multiple Links Failure}

When a node detects a link failure in AODV, it will perform a local recovery algorithm if the failure occurs closer to the destination than to the source node. In this case, node will broadcast a RREQ packet with a limited TTL. If the route is repaired, then the node will forward the DATA packet via the newly discovered route. Otherwise, the node will drop the packet, invalidate the route and send an RERR to the source node to re-initiate a route discovery process. Hence, the delay and overhead in the network will increase.

In contrast, when a node detects a link failure in the RMMMC, it will follow the following procedure: firstly, it will fetch the multi-hop path in its alternative table to the final destination of the packet; then it will look up in its neighbour table for a node in common with the multi-hop path. If any node in the multipath is in its neighbour table, then the node will switch its interface to the home channel of that node and handover the data packet to this node. On the other hand, if none of its neighbours are in the multi-hop alternative path, then the node will drop the data packet and initiate RERR as in AODV protocol.

Figure 6 is an example showing how route recovery in RMMMC is accomplished. When a link break occurs between nodes $M$ and $B$ (see Figure6(a)), node $M$ will check its alternate routing table to $D$. If there is an entry in its alternate routing table to node $D$, then it will compare the accumulated path $\{A, F, Q, S\}$ with its neighbour table. Node $M$ does not find node $A$ in its neighbour table, but finds that node $F$ is in its neighbour table. In this case, node $M$ will update its routing table to $D$ by including node $F$ as a next hop and then forward data to node $F$ (see Figure 6(a)). Therefore, node $M$ uses the alternative route to recover the route rather than dropping the data packet and issuing RERR.

Let us assume there is another link failure between node $F$ and node $A$ (see Figure 6(b)). In this case, node $F$ will check its alternate routing table to $D$. If there is an entry in its alternate table, then it will compare the accumulated paths $\{C, B, M, S\}$ with its neighbour table. Node $F$ finds that node $C$ is in its neighbour table. In this case, node $F$ will update its routing table to $D$ and will then forward the data packet to node $C$. Therefore, the proposed algorithm can handle multiple link failures by re-routing data packets via established routes in different channels. 


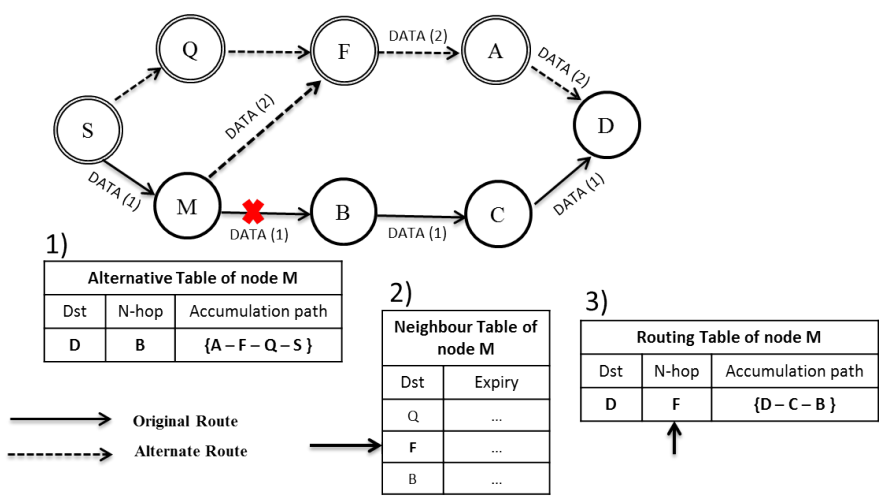

(a)

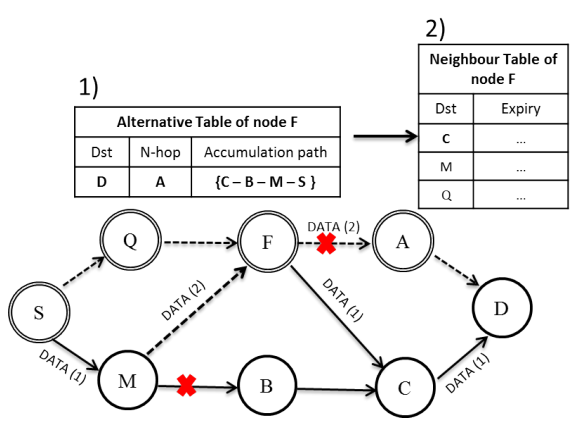

(b)

Fig. 6: Handling link failures in RMMMC.

\section{Simulation PARAMETERS AND PERFORMANCE METRICS}

We evaluated the RMMMC and compared it with the RDT- AODV and AODV protocols using the ns- 2.35 simulator [11]. Table I shows the simulation parameters which were used. We followed the default common parameters in ns-2 for radio power and threshold levels. Source-destination pairs were selected randomly. Each data point in the figures is an average of 9 runs, with a randomly generated topology. Random way-point were used for mobility model. Generally, the 95\% confidence interval was small compared with the value that was being reported.

\section{TABLE I: SIMULATION PARAMETERS}

\begin{tabular}{c|c}
\hline Number of nodes & $50,75,100,125,150,175,200$ \\
\hline Simulation area & $1000 \mathrm{~m} \mathrm{x} 1000 \mathrm{~m}$ \\
\hline Propagation model & Two-ray ground \\
\hline Traffic type & CBR \\
\hline Packet size & 1000 bytes \\
\hline Number of connections & 50 \\
\hline Max speed & 10 \\
\hline Pause time & 20 \\
\hline Generation rate & 2 Packet/s \\
\hline Simulation time & $180(\mathrm{sec})$ \\
\hline
\end{tabular}

The following metrics were used to evaluate the network performance:

Throughput: is the number of data bits successfully delivered to the destination node (measured in Kbps).

Packet delivery ratio (PDR): the ratio of data packets suc-

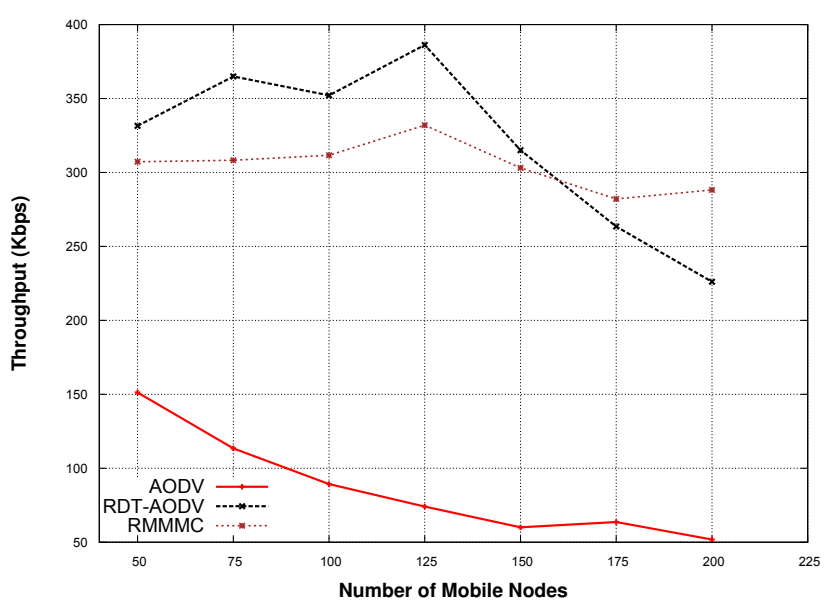

Fig. 7: Throughput.

cessfully received by the destination nodes to the number of data packets generated by the source nodes.

End-to-End Delay (EED): is the average time between transmitting a data packet at the source node and its successful reception at the receiver node (measured in seconds).

\section{Vi. Performance Results}

Figure 7 depict the throughput of the proposed protocol RMMMC compared with the previous work Multi-channel RDT-AODV and the standard single channel AODV with different node densities. Both RDT-AODV and RMMMC achieve better throughput compared with AODV. This is due to the use of multiple channels in communication in both these protocols. RMMMC maintains a steady performance even with the increasing number of nodes. This is may be due the use of a multipath alternative route when link failure occurs. In contrast, the performance of RDT-AODV and AODV drops significantly as the number of nodes increases. This may be due to the number of link failures occurring in high density RDT-AODV and AODV without repairing.

Figure 8 represents the packet delivery ratio for all schemes with various node densities. AODV performance is the lowest with all node densities, compared with RMMMC and RDT-AODV protocols, because of the higher degree of contentions/collisions occurring in a single shared channel. Conversely, RDT-AODV has a better PDR than the RMMMC protocol in low to medium node densities. This may due to the use of a single path in route discovery, which incurs fewer control packets to discover the route. On the other hand, RMMMC outperforms RDT-AODV as the number of nodes increases, as it provides an alternate route when link failure occurs.

Figure 9 shows the average EED for the RMMMC, RDTAODV and AODV protocols with different numbers of nodes. RMMMC and RDT-AODV have less EED compared with that in AODV. This due to the availability of more than one medium for contention, which will reduce the number of collisions occurring in RMMMC and RDT-AODV compared with AODV. However, RMMMC achieves less EED compared 


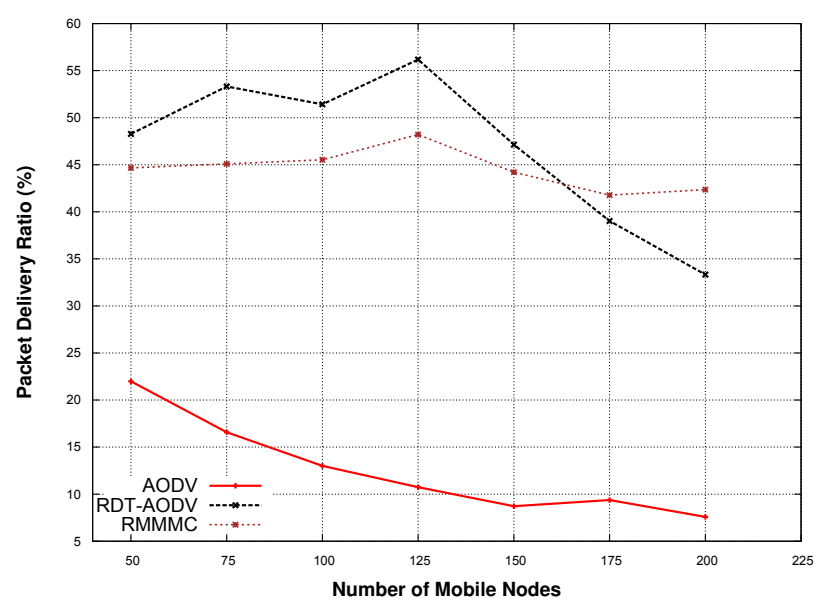

Fig. 8: Packet Delivery Ratio.

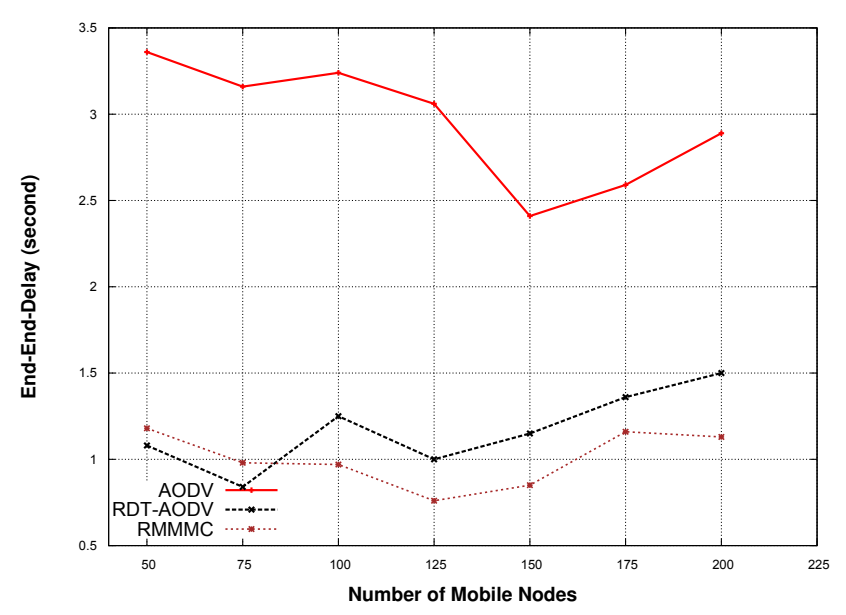

Fig. 9: End-to-End Delay.

with RDT-AODV, as it uses an alternative route in different channel to recover the broken link without re-initiating a new route discovery. This is evident, as the proposed multipath alternative route can reroute the data packet when link failure occurs, if the alternative is available. Hence, the end-to-end delay will be reduced.

\section{CONCLUSIONS AND Future WORK}

In this paper, we have presented a new route discovery mechanism to provide a seamless alternative multi-hop path in a multichannel wireless ad hoc network. The scheme is called Route Migration over Multiple link failure in MultiChannel (RMMMC) wireless ad hoc network with a single radio interface.

RMMMC is a resilient routing protocol that initialises the route discovery mechanisms in multiple paths and announces the availability of any alternative multi-hop path in multiple discovered routes, whenever possible. These paths can be used to repair the failing routes by enquiring from the immediate neighbour in an alternative path to reroute the data packet. Therefore, an immediate route recovery will gained without incurring any extra overhead or delay to repair the failed link. The simulation results showed that the RMMMC protocol achieved better results, as the node densities increased, compared with other approaches.

In our future work, we intend to evaluate RMMMC under different network contexts (high probability of failure and high mobility).

\section{REFERENCES}

[1] C. Perkins, E. Belding-Royer, and S. Das, "Ad hoc on-demand distance vector (aodv) routing," Tech. Rep., 2003.

[2] D. B. Johnson, D. A. Maltz, J. Broch et al., "Dsr: The dynamic source routing protocol for multi-hop wireless ad hoc networks," Ad hoc networking, vol. 5, pp. 139-172, 2001.

[3] I. D. Chakeres and E. M. Belding-Royer, "The utility of hello messages for determining link connectivity," in Wireless Personal Multimedia Communications, 2002. The 5th International Symposium on, vol. 2. IEEE, 2002, pp. 504-508.

[4] I. L. M. S. Committee et al., "Wireless lan medium access control (mac) and physical layer (phy) specifications," IEEE Std, vol. 802, 2007.

[5] N. S. Mirza, M. A. Abdelshafy, and P. J. King, "Performance evaluation of receiver directed transmission protocol with a single transceiver in manets," in Wireless Days, 2017. IEEE, 2017, pp. 241-244.

[6] M. K. Marina and S. R. Das, "On-demand multipath distance vector routing in ad hoc networks," in Network Protocols, 2001. Ninth International Conference on. IEEE, 2001, pp. 14-23.

[7] S.-J. Lee and M. Gerla, "Aodv-br: Backup routing in ad hoc networks," in Wireless Communications and Networking Confernce, 2000. WCNC. 2000 IEEE, vol. 3. IEEE, 2000, pp. 1311-1316.

[8] P. Bahl, R. Chandra, and J. Dunagan, "Ssch: slotted seeded channel hopping for capacity improvement in ieee 802.11 ad-hoc wireless networks," in Proceedings of the 10th annual international conference on Mobile computing and networking. ACM, 2004, pp. 216-230.

[9] J. So and N. H. Vaidya, "A routing protocol for utilizing multiple channels in multi-hop wireless networks with a single transceiver," university of Illinois at Urbana-Champaign, 2004.

[10] L. Shan, L. Xie, Z. Li, and A. Xu, "Partial pre-deflection scheme with least hop first routing for optical burst switching networks under selfsimilar traffic," in Communications and Networking in China, 2006. ChinaCom'06. First International Conference on. IEEE, 2006, pp. 1-5.

[11] “The Network Simulator NS-2, http://www.isi.edu/nsnam/ns/ [retrieved: March, 2017]." 